\title{
Mutation R184Q of connexin 26 in hearing loss patients has a dominant-negative effect on connexin 26 and connexin 30
}

\author{
Ching-Chyuan $\mathrm{Su}^{1}$, Shuan-Yow $\mathrm{Li}^{2,3}$, Mao-Chang Su${ }^{4,5}$, Wei-Chi Chen ${ }^{2}$ and Jiann-Jou Yang ${ }^{\star, 2,3}$
}

Hearing impairment is the most common sensory disorder worldwide. In a recent study, the authors have shown that a heterozygous missense mutation, p.R184Q, in the connexin 26 (Cx26) is causally related to hearing loss. However, the functional change in the Cx26R184Q mutant remains unknown. This study compared the intracellular distribution and assembly of mutant Cx26R184Q with that of the wild-type (WT) Cx26 and Cx30WT in tet-on HeLa cells and the effect that the mutant protein had on those cells. Fluorescent localization assay of WT Cx26 showed the typical punctuate pattern of gap junction channel between neighboring expression cells. Conversely, the p.R184Q missense mutation resulted in accumulation of the Cx26 mutant protein in the Golgi apparatus rather than in the cytoplasmic membrane. Cx26R184Q coexpressed with either Cx26WT or Cx30WT showed perinuclear localization by bidirectional tet-on expression system, suggesting the impairment of the ability of both WT proteins to intracellular trafficking and targeting to the plasma membrane. Therefore, we proposed that Cx26R184Q has a dominant-negative effect on the function of WT Cx26 and Cx30. European Journal of Human Genetics (2010) 18, 1061-1064; doi:10.1038/ejhg.2010.50; published online 5 May 2010

Keywords: Cx26; GJB2; mutation; hearing loss

\section{INTRODUCTION}

Hearing loss, a common sensory disorder in the human population, is often caused by genetic inheritance of auditory system dysfunction. ${ }^{1}$ In developed countries, the estimated incidence of congenital hearing loss is 1 in 1000 births, of which approximately $60 \%$ are attributable to genetic factors. ${ }^{2,3}$ Of the genes responsible for deafness, 59 genes have been identified, some of which are those involved in ion recycling and maintenance. $^{4}$

Connexins (Cxs), a large family of membrane proteins, are key components of gap junctions (GJ). Ions or small molecules pass through these critical intercellular pathways to regulate various physiological and developmental processes. ${ }^{5}$ The GJ channels are double-membrane protein structures that form by head-to-head docking of two half channels, termed connexons, to create hydrophilic pores across the membranes. ${ }^{6}$ Each connexon is composed of six polytopic transmembrane protein subunits, termed connexins. Within a connexon, all Cxs may be identical (homomeric) or different (heteromeric), and two connexons docking together may also be either identical (homotypic junctions) or different (heterotypic junctions). ${ }^{7}$ Other studies have shown that Cxs can assemble into functional hexameric connexons in the endoplasmic reticulum (ER) membrane. ${ }^{8}$ Subcellular fractionation studies and immunocolocalization analyses suggest that $\mathrm{Cxs}$ reach the plasma membrane by passing through the Golgi apparatus. ${ }^{9-11}$
Twenty-one genes have been identified in the human genome coding for connexin proteins. ${ }^{12}$ Recent studies suggested that intercellular communication through GJ is crucial for auditory function. $^{13,14}$ In mice, $\mathrm{Cx} 26$ and $\mathrm{Cx} 30$, apparently the two most abundantly expressed Cxs, are known to co-assemble to form GJ in the cochlea. ${ }^{15}$ Mutations of GJB2, which encodes the Cx26 protein, and GJB6, which encodes the Cx30 protein, are implicated in cases of inherited nonsyndromic hearing loss. ${ }^{1}$ In addition, Cx26 and Cx30 also co-assemble to form heteromeric GJ, and Cx26 mutant protein has dominant-negative effects on Cx30 protein. ${ }^{16}$ In a previous study, the authors identified one heterozygous mutation, c.551G $>$ A (p.R184Q), in the GJB2 gene of a nonsyndromic hearing loss patient. ${ }^{17}$ The family is comprised of a proband with a Cx26 p.R184Q heterozygous variant, a father with hearing loss and a mother with normal hearing. The pedigree strongly suggests that the loss of hearing is inherited in a dominant manner. However, the molecular mechanisms of the mutations leading to hearing loss remain unclear. Therefore, we further investigated this GJB2 gene mutation for mutual correlations with hearing loss. This study used immunofluorescent staining and fusion proteins consisting of Cx with EGFP or DsRed attached to its C terminus to observe the subcellular localization of wild-type (WT) and mutant Cx26 protein in tet-on HeLa cell. In addition, we also investigated the effects of the Cx26R184Q heterozygous mutation protein on WT Cx26 and Cx30 using the tet-on expression system.

\footnotetext{
${ }^{1}$ Tian-Sheng Memorial Hospital, Taiwan, Republic of China; ${ }^{2}$ Department of Biomedical Sciences, Chung Shan Medical University, Taiwan, Republic of China; ${ }^{3}$ Department of Medical Research, Chung Shan Medical University Hospital, Taiwan, Republic of China; ${ }^{4}$ Department of Otorhinolaryngology-Head and Neck Surgery, Chung Shan Medical University Hospital, Taiwan, Republic of China; ${ }^{5}$ Department of Speech Language Pathology \& Audiology and School of Medicine, Chung Shan Medical University, Taiwan, Republic of China

*Correspondence: Dr J-J Yang, Department of Biomedical Sciences, Chung Shan Medical University, No 110, Section 1, Chien-Kun N Road, Taichung 402, Taiwan, Republic of China. Tel: +886 42473 0022, ext. 11804; Fax: +886 42475 7412; E-mail: jiannjou@csmu.edu.tw
}

Received 6 October 2009; revised 4 March 2010; accepted 17 March 2010; published online 5 May 2010 


\section{MATERIALS AND METHODS}

\section{Molecular cloning of wild-type and mutant GJB2 gene}

The mammalian expression vector pcDNA3.1-CT used in this study was constructed as previously described. ${ }^{18}$ We designed and synthesized a tightbinding pair of molecular components comprising a small receptor domain composed of as few as six natural amino acids (-Cys-Cys-Xaa-Xaa-Cys-Cystags) in the C tail of the pcDNA3.1 vector (Invitrogen, Carlsbad, CA, USA). The open reading frames (ORFs) of GJB2 were obtained from genomic DNA by PCR. PCR was carried out with the following oligonucleotide primers: forward primer was GJB2F-HindIII 5'-ATGAAGCTTATGGATTGGGGCACGCTGC-3'

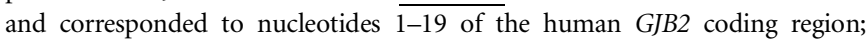
reverse primer was GJB2R-XhoI 5'-ATGGAGCTCGCAACTGGCTTTTTTG ACTTCC- $3^{\prime}$ and corresponded to nucleotides $659-678$ of the human GJB2 coding region (enzyme site nucleotide is underlined). The PCR DNA product (678 bp) of the human GJB2 coding region without the stop codon was cloned into the pcDNA3.1-CT vector. Mutant Cx26 GJ proteins were obtained by performing oligonucleotide-directed mutagenesis using the Stratagene QuikChange Site-Directed Mutagenesis Kit (Stratagene, La Jolla, CA, USA). The following oligonucleotide primers (mutated nucleotide is underlined) were used to prepare the mutant Cx26R184Q gene: Cx26R184Q sense 5'-CTGCTT TGTGTCCCAGCCCACGGAGAAGAC-3'; Cx26R184Q antisense 5'-GTCTTCT CCGTGGGCTGGGACACAAAGCAG-3' ${ }^{\prime}$. For fusion protein generation, cDNA sequences of the autofluorescent reporter proteins EGFP (pEGFPN1 vector; Clontech, Palo Alto, CA, USA) and DsRed (pDsRedN1 vector; Clontech) were fused in-frame to the C terminus of WT and mutant Cx26R184Q. The ORFs of GJB2 were obtained from the pcDNA3.1-CT clone after digestion with HindIII and SacII, and then subcloned into the HindIII and SacII restriction sites in vectors pEGFPN1 and pDsRedN1 (Clontech). The coding region of Cx26WT and that of mutant Cx26R184Q were amplified from plasmids containing the Cx26 cDNA (Cx26wt-EGFP or Cx26R184Q-DsRed) using two pair primers containing recognition sequences $5^{\prime}$-SalI and $3^{\prime}-\mathrm{NotI}$ or $5^{\prime}-\mathrm{NheI}$ and $3^{\prime}-E c o \mathrm{RV}$, respectively, and Platinum Pfx DNA Polymerase (Invitrogen). Purified products were subcloned into the corresponding site of the bidirectional expression vector pBI (Clontech). The dideoxy DNA sequencing method, using a DNA sequencing kit (Applied Biosystems Corporation, Foster City, CA, USA), an ABI Prism 3730 Genetic Analyzer (Applied Biosystems Corporation) and restriction digest were used to confirm the DNA sequence of all constructs.

\section{Transfection and expression of Cx26WT, Cx26R184Q, Cx26WT/Cx26R184Q and Cx30WT/Cx26R184Q chimerae protein} in tet-on HeLa cell line

The tet-on HeLa cell line deficient in the GJIC gene was purchased from BD Biosciences Clontech and maintained in Dulbecco's modified Eagle's medium, supplemented with 10\% FBS (Gibco BRL, Gaithersburg, MD, USA), $100 \mu \mathrm{g} / \mathrm{ml}$ G418, $100 \mathrm{U} / \mathrm{ml}$ penicillin and $100 \mu \mathrm{g} / \mathrm{ml}$ streptomycin at $37^{\circ} \mathrm{C}$ in a moist atmosphere containing $5 \% \mathrm{CO}_{2}$. Transfection was carried out using the LipofectAMINE reagent (Invitrogen) according to the manufacturer's instructions. A ratio of $1 \mu \mathrm{g}$ DNA $v s 2 \mu$ l LipofectAMINE 2000 was used for the tet-on $\mathrm{HeLa}$ cells. Cells were harvested at $24 \mathrm{~h}$ after transfection and grown on a coverslip for $24 \mathrm{~h}$ at $37^{\circ} \mathrm{C}$ in a humidified $5 \% \mathrm{CO}_{2}$ incubator. Then, tet-on HeLa cells were treated with $1 \mu \mathrm{g} / \mathrm{ml}$ doxycyclin (Dox; Sigma-Aldrich Corporation, St Louis, MO, USA) in cell culture medium to induce Cx26WT, Cx30WT, Cx26R184Q or both WT and mutant protein expressions.

Immunofluorescence staining of post-transfection tet-on HeLa cells Wild-type or mutant Cx protein expression in tet-on HeLa cells was analyzed by a direct fluorescent protein fusion method involving fusion of EGFP or DsRed to the C-terminal ends of the Cx proteins. Briefly, post-transfection tet-on HeLa cells grown on coverslips were exposed to Dox for $5 \mathrm{~h}$ before immunofluorescence staining. Tet-on HeLa cells were fixed with $4 \%$ paraformaldehyde in $0.1 \mathrm{M}$ PBS for $20 \mathrm{~min}$ and then rinsed three times in PBS. Then, the coverslips were immersed in $10 \%$ normal goat serum and $0.1 \%$ Triton $\mathrm{X}-100$ for $15 \mathrm{~min}$. The primary antisera and dilutions were as follows: mouse antipan-cadherin antibody at 1:200 (anti-CH19; Abcam plc., Cambridge, UK) for cell membrane and mouse anti-Golgin-97 at 1:200 (Invitrogen) for Golgi apparatus. After incubation with primary antiserum at $4{ }^{\circ} \mathrm{C}$ overnight, the cells were rinsed in PBS three times before adding Alexa Fluor 488- and/or Alexa Fluor 594-conjugated secondary antibodies (Invitrogen). ER was stained with ER-Tracker Blue-white DPX Probes at 1:670 dilution (Invitrogen) for $10 \mathrm{~min}$ at room temperature. The nuclei of cells were counterstained with 4'-6-diamidino-2phenylindole (DAPI; $2 \mu \mathrm{g} / \mathrm{ml}$ ) or propidium iodide (PI; $1 \mathrm{mg} / \mathrm{ml} ; 1: 400$ dilution) for $5 \mathrm{~min}$ and rinsed with PBS. Mounted slides were visualized and photographed using a fluorescence microscope (Zeiss Axioplam, Oberkochen, Germany).

\section{RESULTS}

Cx26 consists of four transmembrane domains linked to the cytoplasmic C- and N-terminal ends by one cytoplasmic and two extracellular loops. The p.R184Q substitutions were detected in the second extracellular loop (E2) of the Cx26 protein. To elucidate the effect of the p.R184Q missense mutation, we examined amino-acid sequences of Cx26 using ConSeq analysis (http://conseq.tau.ac.il/). After depositing the protein sequence of $\mathrm{Cx} 26$ into the system, it automatically collected homologous sequences of $\mathrm{Cx} 26$ and constructed multiple sequence alignments. The system collected 114 PSI-BLAST hits, of which 96 were unique sequences. The system then automatically calculated the 50 sequences with the lowest $E$-values. The sequencing results revealed that p.R184 is highly conserved (Conseq score $=8-9$ ), which is a predicted functional residue and exposed outside the protein (Supplementary Figure S1).

To elucidate how p.R184Q affects the functional properties and subcellular localization of the Cx26 protein, this study used lipofection to transiently transfect GJ-deficient tet-on HeLa cells with cDNA constructs of WT or mutant Cx26. In cells transiently expressing Cx26WT-EGFP, Cx26-EGFP expression was observed localized to the cell membranes at points of contact between adjacent EGFPexpressing cells, indicating the formation of GJ plaques (Figure 1a). This membrane localization was confirmed by colocalization with
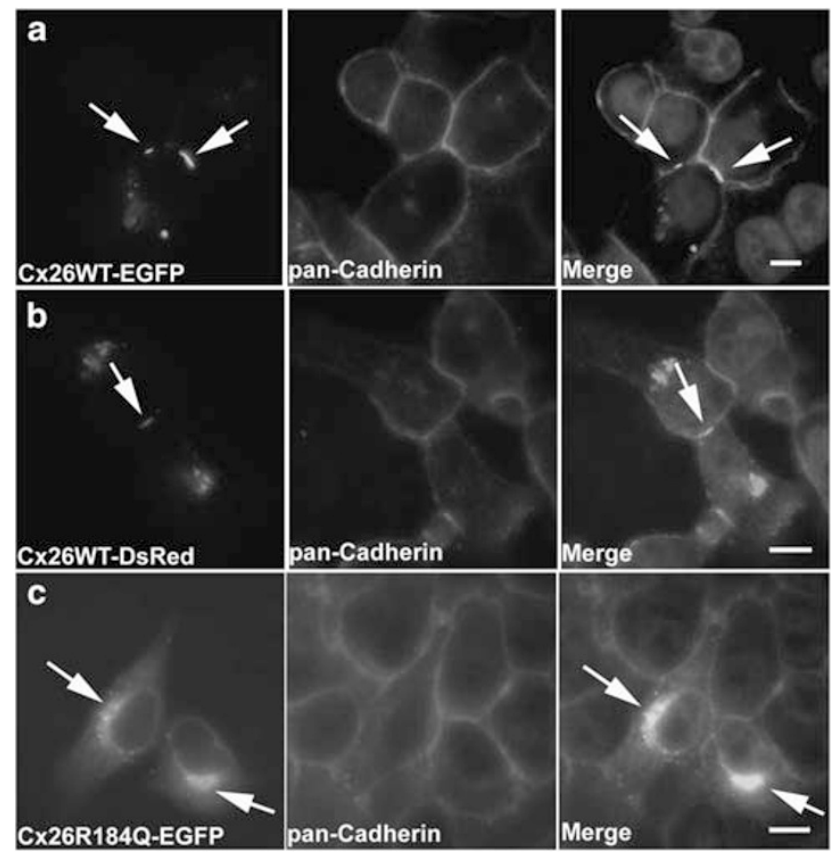

Figure 1 Analysis of Cx26WT and Cx26R184Q expression in transiently transfected tet-on HeLa cells by immunocytochemistry using pan-cadherin antibody. Fluorescence microscopy of Cx26WT-EGFP (a) and Cx26WT-DsRed (b) tet-on HeLa cells showing expression of Cx26 fusion protein in the plasma membranes. The CX26R184Q-EGFP (c) transfected HeLa cells show impaired trafficking and concentration near the nucleus. Cells were counterstained with 4'-6-diamidino-2-phenylindole (DAPI) to highlight the nuclei. Arrows indicate the localization of $\mathrm{C} \times 26$ protein. Scale bars $=10 \mu \mathrm{m}$. 


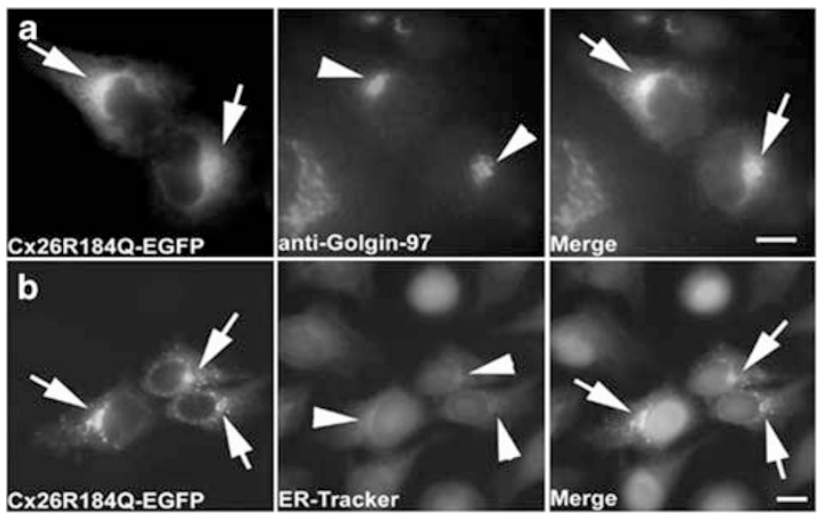

Figure 2 Intercellular localization of the mutant Cx26R184Q protein. Photomicrographs of tet-on HeLa cells transiently transfected with Cx26R184QEGFP cDNA and immunostained for use as markers of Golgi apparatus (a) and endoplasmic reticulum (ER) (b), respectively. The staining results for mutant Cx26R184Q showed substantial colocalization in the Golgi apparatus and slight colocalization with ER marker. The cells were counterstained with 4'-6diamidino-2-phenylindole (DAPI) or propidium iodide (PI) to highlight the nuclei. Arrows indicate the localization of Cx26 mutant protein. Arrowheads indicate the localization of Golgi apparatus or ER. Scale bars $=10 \mu \mathrm{m}$.

pan-Cadherin (Figure 1a). Similarly, Cx26WT-DsRed also localized to the cell membrane (Figure 1b). However, Cx26R184Q-EGFP mutant protein was impaired in trafficking to the cell membrane and then consequently concentrated in the cytoplasm close to the nucleus (Figure 1c). This study then investigated which organelles in the cytoplasm of the mutant Cx26 localized in tet-on HeLa cells that had been transfected with Cx26R184Q cDNA were immunostained with markers for Golgi apparatus and ER (Figure 2). The analytical results indicated that most accumulation of p.R184Q of Cx26 mutant protein was in the Golgi apparatus (Figure 2a). Some proteins showed a reticular pattern and were colocalized with ER-tracker Blue-white DPX probe, an ER marker (Figure $2 b$ ).

Our previous study found that p.R184Q of Cx26 is a heterozygous mutation in a hearing loss patient. ${ }^{17}$ Consequently, coexpression studies were performed to examine the effects of the mutant proteins on Cx26WT in tet-on HeLa cell using the bidirectional tet-on protein expression system with equal amounts of the two respective expression proteins. Cells expressing both Cx26WT-DsRed and the Cx26WTEGFP protein showed co-assembly expression in the cell membrane of tet-on HeLa cells (Figure 3a). Conversely, both Cx26WT-DsRed and the Cx26R184Q-EGFP mutant revealed expression patterns resembling those of p.R184Q alone (Figure 3b). Analytical results indicated that Cx26R184Q mutant protein showed co-assembly with Cx26WT protein and impaired Cx26WT protein trafficking to the cell membrane.

Reports of Cx26 and Cx30 coexpression in the inner ear ${ }^{1,16,19}$ prompted us to examine the effects of the mutant Cx26R184Q protein on Cx30WT using the tet-on protein expression system. Both Cx26WT-DsRed and Cx30WT-EGFP were colocalized at the points of contact between adjacent tet-on HeLa cells (Figure 4a). This showed that both Cx26 and Cx30 can be trafficked to the same GJ plaque. However, coexpression of Cx26R184Q-EGFP and Cx30WT-DsRed produced a localization change of these $\mathrm{Cx} 30 \mathrm{WT}$ proteins from the cell membrane to concentrate in the cytoplasm (Figure 4b; Supplementary Figure S2). Analytical results indicated that Cx26R184Q mutant protein showed coassembly with Cx30WT protein and impaired Cx30WT protein trafficking to the cell membrane.

As a result, we propose that p.R184Q of Cx26 has a dominantnegative effect on Cx26WT and Cx30WT.

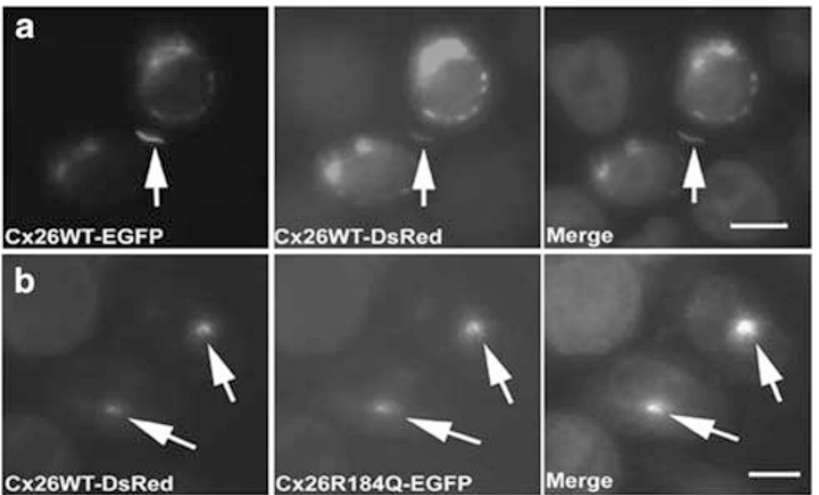

Figure 3 Coexpression of mutant proteins with Cx26WT by the tet-on protein expression system. (a) Tet-on HeLa cells coexpressing Cx26WT-DsRed and Cx26WT-EGFP, revealing colocalization of the two proteins at the plasma membrane. (b) Tet-on HeLa cells coexpressing Cx26WT-DsRed and Cx26R184-EGFP revealing colocalization of the two proteins around the nucleus regions. Arrows indicate coexpressed protein. The cells were counterstained with 4'-6-diamidino-2-phenylindole (DAPI) to highlight the nuclei. Scale bars $=10 \mu \mathrm{m}$.

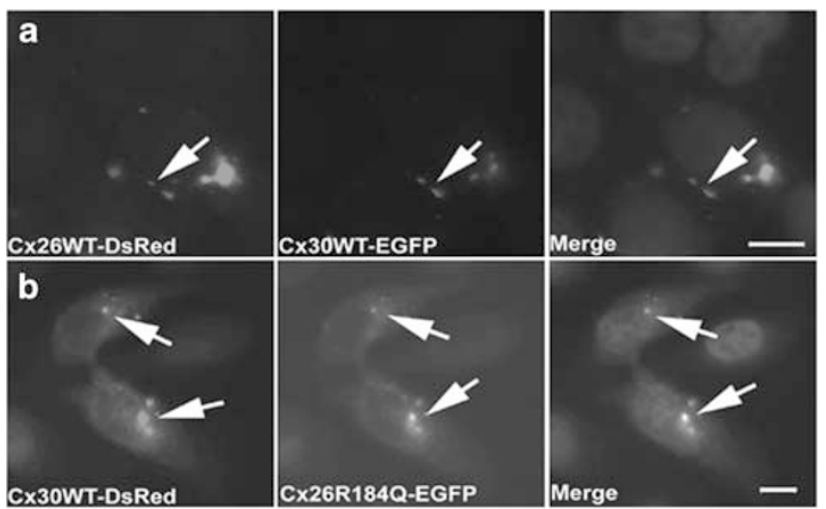

Figure 4 Coexpression of mutant proteins with Cx30WT by the tet-on protein expression system. (a) Tet-on HeLa cells coexpressing Cx26WT-DsRed and Cx30WT-EGFP revealing colocalization of the two proteins at the plasma membrane. (b) Tet-on HeLa cells coexpressing Cx30WT-DsRed and Cx26R184-EGFP revealing colocalization of the two proteins around the nucleus regions. Arrows indicate coexpressed protein. The cells were counterstained with 4'-6-diamidino-2-phenylindole (DAPI) to highlight the nuclei. Scale bars $=10 \mu \mathrm{m}$.

\section{DISCUSSION}

Functional studies of Cx expression ${ }^{20}$ have reconstituted GJ communication in transfected mammalian cells (eg, HeLa cell). Coexpressed fluorescent reporter and expressed fluorescent-protein-tagged (eg, green fluorescent protein, GFP) have also been used for rapid and efficient functional analysis of several Cxs, including Cx26, Cx30, Cx31 and $\mathrm{Cx} 43 .{ }^{20-23}$ The tet-on protein expression system, a high-level gene expression system, was first described by Gossen et al. ${ }^{24}$ The bidirectional (pBI) tet-on expression vectors are specially designed response vectors that allow coregulated expression of two genes under control of a single tet response element. The pBI vector can induce equal amounts of the two expression proteins under the regulation of doxycycline. We therefore studied the subcellular localization of the heterozygous mutant $\mathrm{Cx} 26$ protein that was expressed by using a combination of tet-on HeLa cells, the bidirect tet-on protein expression system and expressed fluorescent-tagged protein. 
Mutation of the GJB2 gene encoding Cx26 protein is the most common cause of hereditary, prelingual and nonsyndromic hearing loss. ${ }^{1}$ Approximately 90 GJB2 mutations causing abnormal Cx26 expression have been reported and linked to hearing impairment. ${ }^{25}$ The p.R184Q (551G $>$ A) was also described elsewhere ${ }^{26}$ and in our earlier study in Taiwan. ${ }^{17}$ Position 184 of GJB2 is located at the second extracellular loop (E2). The R184Q of GJB2 at E2 might result in incompatibility between different species of connexin proteins to form heterotypic functional channels. ${ }^{27}$ ConSeq is a web site server for identifying biologically important residues in protein sequences. ${ }^{28}$ The ConSeq indicated that p.R184 of Cx26 is exposed, highly conserved and throughout evolutionary. Two other missense mutations (R184W and R184P) at the same position have been associated with hearing impairments. ${ }^{29,30}$ We also found that the p.R184Q missense mutation of Cx26 causes Cx26 mutant protein accumulation in the Golgi apparatus instead of targeting to cytoplasmic membrane. Finally, according to our results, Cx26R184Q have dominant-negative effect on the function of WT Cx26 and Cx30 in the co-expression study using bidirect tet-on protein expression system. Therefore, we suggest that the p.R184 amino acid is perhaps critical in Cx26, and as a result a mutation in this residue will lead to loss of function of the protein.

Generally, Cxs are translated in the ER and then posttranslationally modified and packaged into the membrane vesicles of the Golgi. They reach the cortical plasma membrane along microtubules as tracks and then diffuse laterally within the plasma membrane before finally residing at cell-to-cell contact regions. ${ }^{31,32} \mathrm{~A}$ recent study indicated that $\mathrm{Cx} 26$ and $\mathrm{Cx} 30$ travel to the plasma membrane by bypassing the Golgi apparatus (Golgi-independent) secretory route. The route differs from the classical secretory route of $\mathrm{Cx} 32$ and $\mathrm{Cx} 43 .{ }^{32}$ The analytical results in this study revealed heavy and moderate accumulations of Cx26 mutant protein in the Golgi apparatus and ER, respectively. The presumed explanation is that when Cx26R184Q mutations occur, the Cx26 mutant protein trafficking route was altered, subsequently causing accumulation in the Golgi apparatus. Another possibility is that the Cx26R184Q mutant protein impairs trafficking by changing its 3D structure. Cx26R184Q mutant proteins were observed in the ER, suggesting that it is a newly synthesized protein. In addition, $\mathrm{Cx} 26$ and $\mathrm{Cx} 30$ share a similar protein structure and the same pattern of expression in the cochlea in the form of homotypic or heterotypic channels. The trafficking and assembly features of Cx30 are also similar to those of Cx $26 .{ }^{32}$ These properties facilitate the assembly of heterotypic GJs and help explain the dominant-negative effect of WT Cx30 by Cx26R184Q. However, the detailed mechanisms and components of the molecular machinery that mediate this unconventional process remain unclear.

Therefore, further study is warranted to clarify the influence of this mutation on the protein level and 3D structure of WT and mutant $\mathrm{Cx} 26$ protein. Sucrose gradient analysis and immunoprecipitation are also suggested for further confirmation and to resolve the interesting question of the Cxs ratio in individual connexons and the trafficking machinery of Cx26R184Q mutation protein.

\section{CONFLICT OF INTEREST}

The authors declare no conflict of interest.

\section{ACKNOWLEDGEMENTS}

We thank the Chung Shan Medical University, Tian-Sheng Memorial Hospital (CSMU-TSMH-097-001) and the National Science Council of the
Republic of China, Taiwan for financially supporting this research under Contract No. NSC98-2320-B-040-016-MY3. Ted Knoy is appreciated for the editorial assistance.

1 Apps SA, Rankin WA, Kurmis AP: Connexin26 mutations in autosomal recessive deafness disorders: a review. Int J Audiol 2007; 46: 75-81.

2 Morton NE: Genetic epidemiology of hearing impairment. Ann NYAcad Sci 1991; 630: 16-31.

3 Pallares-Ruiz N, Blanchet P, Mondain M, Claustres M, Roux AF: A large deletion including most of GJB6 in recessive nonsyndromic deafness: a digenic effect? Eur J Hum Genet 2002; 10: 72-76.

4 Morton CC: Genetics, genomics and gene discovery in the auditory system. Hum Mol Genet 2002; 11: 1229-1240.

5 Evans WH, Martin PE: Gap junctions: structure and function (review). Mol Membr Biol 2002; 19: 121-136.

6 Makowski L, Caspar DLD, Phillips WC, Goodenough DA: Gap junction structures. J Cell Biol 1997; 74: 629-645.

7 Willecke K, Eiberger J, Degen J et al: Structural and functional diversity of connexin genes in the mouse and human genome. Biol Chem 2002; 383: 725-737.

8 Falk MM, Buehler LK, Kumar NM, Gilula NB: Cell-free synthesis of connexins into functional gap junction membrane channels. EMBO J 1997; 10: 2703-2716.

9 Musil LS, Goodenough DA: Biochemical analysis of connexin43 intracellular transport, phosphorylation, and assembly into gap junction plaques. J Cell Biol 1991; 115: 1357-1374.

10 Falk MM, Kumar NM, Gilula NB: Membrane insertion of gap junction connexins: polytopic channel forming membrane proteins. J Cell Biol 1994; 127: 343-355.

11 Laird DW, Castillo M, Kasprzak L: Gap junction turnover, intracellular trafficking, and phosphorylation of connexin43 in brefeldin A-treated rat mammary tumor cells. J Cell Biol 1995; 131: 1193-1203.

12 Sohl G, Willecke K: Gap junctions and the connexin protein family. Cardiovasc Res 2004; 62: 228-232.

13 Zhao HB, Kikuchi T, Ngezahayo A, White TW: Gap junctions and cochlear homeostasis. J Membr Biol 2006; 209: 177-186.

14 Kikuchi T, Kimura RS, Paul DL, Takasaka T, Adams JC: Gap junction systems in the mammalian cochlea. Brain Res 2000; 32: 163-166.

15 Ahmad S, Chen S, Sun J, Lin X: Connexins 26 and 30 are co-assembled to form gap junctions in the cochlea of mouse. Bioche Biophy Res Comm 2003; 307: 362-368.

16 Marziano NK, Casalotti SO, Portelli AE, Becker DL, Forge A: Mutations in the gene for connexin 26 (GJB2) that cause hearing loss have a dominant negative effect on connexin 30. Hum Mol Genet 2003; 12: 805-812.

17 Yang JJ, Huang SH, Chou KH, Liao PJ, Su CC, Li SY: Identification of mutations in members of connexin gene family as a cause of nonsyndromic deafness in Taiwan. Audiol Neurootol 2007; 12: 198-208.

18 Griffin BA, Adams SR, Tsien RY: Specific covalent labeling of recombinant protein molecules inside live cells. Science 1998; 281: 269.

19 Lautermann J, ten Cate WJ, Altenhoff P et al: Expression of the gap-junction connexins 26 and 30 in the rat cochlea. Cell Tissue Res 1998; 294: 415-420.

20 Beltramello M, Bicego M, Piazza V, Ciubotaru CD, Mammano F, D'Andrea P: Permeability and gating properties of human connexins 26 and 30 expressed in HeLa cells. Biochem Biophys Res Comm 2003; 305: 1024-1033.

21 D'Andrea P, Veronesi V, Bicego $M$ et al: Hearing loss: frequency and functional studies of the most common connexin26 alleles. Biochem Biophys Res Commun 2002; 296: 685-691.

22 Common JE, Becker D, Di WL, Leigh IM, O'Toole EA, Kelsell DP: Functional studies of human skin disease- and deafness-associated connexin 30 mutations. Biochem Biophys Res Commun 2002; 298: 651-656.

23 Thomas T, Jordan K, Simek J et al: Mechanisms of Cx43 and Cx26 transport to the plasma membrane and gap junction regeneration. J Cell Sci 2005; 118: 4451-4462.

24 Gossen M, Freundlieb S, Bender G, Muller G, Hillen W, Bujard H: Transcriptional activation by tetracycline in mammalian cells. Science 1995; 268: 1766-1769.

25 Azaiez H, Chamberlin GP, Fischer SM et al: GJB2: the spectrum of deafness-causing allele variants and their phenotype. Hum Mutat 2004; 24: 305-311.

26 Hamelmann C, Amedofu GK, Albrecht K et al: Pattern of connexin 26 (GJB2) mutations causing sensorineural hearing impairment in Ghana. Hum Mutat 2001; 18: 84-85.

27 Krutovskikh V, Yamasaki H: Connexin gene mutations in human genetic diseases. Muta Res 2000; 462: 197-207.

28 Berezin C, Glaser F, Rosenberg J et al: ConSeq: the identification of functionally and structurally important residues in protein sequences. Bioinformatics 2004; 20: 1322-1324.

29 Wilcox SA, Saunders K, Osborn AH et al: High frequency hearing loss correlated with mutations in the GJB2 gene. Hum Genet 2000; 106: 399-405.

30 Denoyelle F, Weil D, Maw MA et al: Prelingual deafness: high prevalence of a 30delG mutation in the connexin 26 gene. Hum Mol Genet 1997; 6: 2173-2177.

31 Evans WH, Ahmad S, Diez J, George CH, Kendall JM, Martin PE: Trafficking pathways leading to the formation of gap junctions. Novartis Found Symp 1999; 219: 44-54.

32 Qu C, Gardner P, Schrijver I: The role of the cytoskeleton in the formation of gap junctions by connexin 30. Exp Cell Res 2009; 315: 1683-1692.

Supplementary Information accompanies the paper on European Journal of Human Genetics website (http://www.nature.com/ejhg) 